\title{
Estresse oxidativo no plasma sanguíneo de indivíduos submetidos ao esforço físico agudo seguido de crioimersão corporal
}

\author{
Oxidative stress in blood plasma in persons who are submitted to cold water \\ immersion after acute physical exercise
}

Fernanda Peres Prado', Deise Regina Barbosa Paludetto', Cynthia Antonia Kallas Bachur ${ }^{2}$, Roseli Aparecida Leite de Freitas ${ }^{2}$, José Eduardo Zaia ${ }^{3}$, Turíbio Leite Barros Neto ${ }^{4}$, Sérgio Britto Garcia ${ }^{5}$, Ana Carolina Araruna Alves ${ }^{6}$, Paulo Tarso Camilo de Carvalho7, José Alexandre Bachur ${ }^{2}$

\begin{abstract}
RESUMO I O objetivo deste estudo foi analisar a influência da crioimersão corporal (CIC) imediata ao esforço físico agudo no estresse oxidativo (EOx) no plasma sanguíneo. Participaram do presente estudo 12 homens, com idade média de $22 \pm 1$ anos, submetidos ao teste de esforço físico intenso em esteira, seguido de $\mathrm{ClC}$ em um tanque com água a $10^{\circ} \mathrm{C}$ durante 10 minutos contínuos. Do repouso ao final da $\mathrm{CIC}$, os indivíduos foram monitorados através de alguns parâmetros como: o índice de percepção subjetiva do esforço (IPE) expresso conforme escala de Borg, frequência cardíaca (FC), pressão arterial (PA) e temperatura corporal (TC) através da temperatura timpânica. A análise morfológica do EOx plasmático foi realizada de acordo com o método denominado Morfologia Óptica do Estresse Oxidativo no Plasma (MEOP), utilizando-se gotas de sangue capilar. Observou-se uma significativa elevação $(p<0,01)$ no grau do estresse oxidativo plasmático após a realização do esforço físico, em relação ao respectivo grau em repouso. Porém, esta elevação no grau do EOx foi significativamente reduzida $(p<0,001)$ em função da CIC. Embora sejam necessários mais estudos científicos com o MEOP, concluiu-se que, para o presente estudo, este teste mostrou-se viável. Os dados encontrados no presente estudo sugerem que a $\mathrm{ClC}$ em água a $10^{\circ} \mathrm{C}$ por 10 minutos imediatos ao esforço físico agudo com intensidade alta, apresenta-se como uma importante conduta fisioterapêutica para a normalização do EOx pós-esforço.
\end{abstract}

Descritores I exercício; estresse oxidativo; radicais livres; crioterapia; imersão.

\begin{abstract}
I The purpose of this study was to analyze the influence of cold water immersion (CWI) following acute physical exercise on the oxidative stress in the blood plasma. Twelve men with average age of $22 \pm 1$ years old, participated this study. All of them underwent the treadmill stress test followed by CWl in tank with water at $10^{\circ} \mathrm{C}$ for 10 minutes. During this process, they were monitored and a set of parameters were analyzed: physical effort perception (Borg Scale), Heart Rate (HR), Blood Pressure (BP) and body heat, by measuring tympanic temperature. The morphological analysis of oxidative stress in blood plasma was done in accordance with a method known as Optical Morphology of the Oxidative Stress on Blood Plasma, which uses drops of capillary blood. A significant increase $(p<0.01)$ in the rate of oxidative stress in plasma was noted after intense physical effort when compared with the rate of stress while at rest. However, the stress rate was significantly decreased $(p<0.001)$ after $\mathrm{CWI}$. Although further scientific studies should be carried out on the above mentioned test, may be concluded that, in the present study, the test has proven itself viable. The data found in the present study suggest that cold water immersion at $10^{\circ} \mathrm{C}$ for 10 minutes following high intensity physical effort appears to be an important physical therapeutic measure towards the normalization of oxidative stress.
\end{abstract}

Keywords I exercise; oxidative stress; free radicals;

cryotherapy; immersion.

\footnotetext{
Estudo desenvolvido no Laboratório de Estudos da Fadiga e Recuperação do Equilíbrio Orgânico da Universidade de Franca (UNIFRAN) - Franca (SP), Brasil.

'Fisioterapeuta graduada e pós-graduada em Fisioterapia pela UNIFRAN - Franca (SP), Brasil.

${ }^{2}$ Professor Pesquisador do Curso de Fisioterapia da UNIFRAN - Franca (SP), Brasil.

${ }^{3}$ Professor do Programa de Doutorado e Mestrado em Promoção de Saúde da UNIFRAN - Franca (SP), Brasil.

${ }^{4}$ Professor da Universidade Federal de São Paulo (UNIFESP) - São Paulo (SP), Brasil.

${ }^{5}$ Docente da Faculdade de Medicina de Ribeirão Preto da Universidade de São Paulo (USP) - Ribeirão Preto (SP), Brasil.

FFisioterapeuta; Aluna do Programa de Doutorado e Mestrado em Ciências da Reabilitação da Universidade Nove de Julho (UNINOVE) São Paulo (SP), Brasil.

${ }^{7}$ Professor do Programa de Doutorado e Mestrado em Ciências da Reabilitação da UNINOVE - São Paulo (SP), Brasil.
} 


\section{INTRODUÇÃO}

A prática regular de exercício, tem sido amplamente associada à melhoria dos padrões de saúde ${ }^{1,2}$, por diminuir a morbidade e consequentemente a mortalidade ${ }^{3,4}$, favorecendo a adequação da composição corporal ${ }^{5}$, as funções cardiovasculares e pulmonares, a circulação periférica e as atividades musculares esqueléticas ${ }^{6}$, dentre outros.

No entanto, o exercício físico agudo exaustivo, pode provocar alterações orgânicas nocivas, especialmente no organismo não adaptado às alterações homeostásicas impostas pelo esforço realizado ${ }^{3}$. Podem ser observados distúrbios fisiológicos e metabólicos associados a um aumento súbito do fluxo do oxigênio mitocondrial, e a subsequente formação das espécies reativas do oxigênio $(\mathrm{EROS})^{7-9}$.

O estresse oxidativo (EOx) decorrente do desequilíbrio entre a quantidade de EROS formada e a biodisponibilidade de antioxidantes ${ }^{10}$, configura-se em uma sobrecarga oxidativa com magnitude dependente da intensidade e duração do exercício realizado ${ }^{11}$, podendo estar associado à fadiga, à redução da performance e ao dano muscular ${ }^{7}$, devido às lesões oxidativas junto aos lipídios, ácidos nucleicos e proteínas celulares ${ }^{12}$.

A recuperação pós-esforço fatigante tem sido benéfica e sua otimização se torna a cada dia significativamente importante para a manutenção ou melhoria do desempenho físico. Uma recuperação adequada está relacionada a um conjunto de ocorrências associadas entre si, como: a diminuição da fadiga, a aceleração da regeneração fisiológica, o realce da adaptação e (possivelmente) a diminuição do risco do ferimento tecidual, se caracterizando por neutralizar os efeitos deletérios subsequentes aos estímulos excedentes ocorridos durante o esforço físico e, por permitir que ocorra o retorno dos tecidos ao seu estado pré-exercício, reabastecendo-se das moléculas de energia associado à remoção dos metabólitos. Nos últimos anos, a imersão em água gelada ou crioimersão corporal (CIC) se tornou uma das estratégias de recuperação mais utilizadas, embora ainda haja uma significativa carência de estudos esclarecedores quanto aos seus mecanismos moleculares de ação ${ }^{13,14}$.

Benefícios decorrentes recuperação pós-esforço promovida pela CIC têm sido comumente associados à pressão hidrostática, visto que o corpo imerso sofre uma força compressiva provocada pela água ambiente, que resulta na movimentação do fluído extracapilar para o meio intravascular em direção ao compartimento corporal central, promovendo uma importante elevação na taxa de remoção dos resíduos metabólicos associada a uma proporcional mobilização e distribuição de substratos ${ }^{14}$. Além destes aspectos microcirculatórios, outro fator importante associado à CIC tem sido a redução da temperatura corporal e a consequente diminuição do metabolismo celular ${ }^{15}$.

\section{OBJETIVO}

Analisar a influência da CIC imediata ao esforço físico agudo no EOx no plasma sanguíneo.

\section{METODOLOGIA}

No presente estudo, aprovado pelo Comitê de Ética em Pesquisa com Seres Humanos da Universidade de Franca (041/009), os participantes concordaram e assinaram o termo de consentimento livre e esclarecido elaborado conforme a Resolução no 196/96 do Conselho Nacional de Saúde.

A amostra estudada foi composta de 12 indivíduos inclusos com base nos seguintes critérios: sexo masculino, sadios, com idade média de $22 \pm 1$ anos, não tabagistas, não usuários de drogas e de álcool, fisicamente ativos e cognitivamente aptos à realização do esforço físico proposto, com valor médio do índice de massa corpórea (IMC) mais ou menos o valor do erro padrão da média igual a $24 \pm 1 \mathrm{~kg} / \mathrm{m}^{2}$. Os parâmetros, índice de percepção do esforço físico (IPE), a frequência cardíaca (FC), a pressão arterial (PA) e a temperatura corporal (TC) foram monitorados para maior segurança dos indivíduos em relação à realização do esforço físico assim como também para se confirmar o grau de uniformidade do grupo amostral do presente trabalho nos diferentes momentos de estudo preestabelecidos: em repouso, no esforço físico (a cada quatro minutos desde o início), ao final da CIC e no décimo minuto pós-crioimersão.

Enquanto o apontamento do IPE foi feito de acordo com a Escala de Borg de 0 a $10^{16}$, o monitoramento da FC foi realizado mediante a utilização do frequencímetro Polar A3 acoplado ao tórax do indivíduo. Para a aferição da PA, foram utilizados o esfigmomanômetro de coluna de mercúrio previamente calibrado e um estetoscópio (Rappaport) para adultos. A TC foi monitorada com base na temperatura timpânica, a qual tem sido considerada a mais fidedigna representante da TC central. Para tanto, a extremidade termossensível do termômetro timpânico (Thermoscan) foi suavemente 
introduzida no conduto auditivo em direção à membrana timpânica. Mediante a emissão automática do alerta sonoro, o referido termômetro foi imediatamente retirado para a devida leitura do valor da temperatura aferida expresso em graus Celsius $\left({ }^{\circ} \mathrm{C}\right)$.

O esforço físico agudo foi realizado sobre uma esteira rolante elétrica com inclinação fixa sob condições ambientais adequadas e controladas $\left(23^{\circ} \mathrm{C}\right)$. Os indivíduos iniciaram o teste a partir de uma velocidade de $2,0 \mathrm{Km} / \mathrm{h}$ e a intensidade do esforço físico foi determinada pelo implemento progressivo da velocidade de 1 $\mathrm{km} / \mathrm{h}$ a cada 4 minutos, até o indivíduo atingir uma FC relativa a $85 \%$ da frequência máxima predita através da fórmula de Karvonen. A partir deste momento, o indivíduo continuou a realização do exercício em uma velocidade constante até atingir o índice de percepção da sua máxima capacidade de dar continuidade ao referido esforço (IPE=10 pontos).

Após a realização do referido esforço físico agudo, todos os indivíduos foram submetidos ao procedimento de imersão corporal até a região xifoidiana, em um tanque de inox (de $80 \mathrm{~cm}$ de altura por $60 \mathrm{~cm}$ de largura e $100 \mathrm{~cm}$ de comprimento) com água a $10^{\circ} \mathrm{C}$ durante 10 minutos conforme o Protocolo de $\mathrm{CIC}^{17}$.

A avaliação morfológica do EOx no plasma sanguíneo foi realizada de acordo com o teste denominado de Microscopia Ótica para a Análise Celular In Vitro ou Teste HLB, também conhecido sob a denominação de Dry layer oxidative test, ao qual nos referimos como morfologia microscópica do EOx no plasma (MEOP).

O MEOP proporciona informações indiretas relativas ao EOx, dentre outras, utilizando-se de seguinte sistema de graduação do percentual da área descontínua do coágulo, presente na lâmina de microscopia: grau I (taxa de EOx normal, com área total de descontinuidade da matriz extracelular inferior a 10\%), grau II (EOx leve, com área de descontinuidade da ME entre $10 \mathrm{a}$ 20\%), grau III (EOx moderado, com área de descontinuidade da ME entre 20 a 30\%), grau IV (EOx grave, com área de descontinuidade da $\mathrm{ME}$ entre 30 a 40\%) e, o grau V (EOx muito grave, com área de descontinuidade da ME acima de 40\%) ${ }^{18}$.

Mediante a punção capilar na face medial da polpa digital do quarto dedo da mão esquerda previamente higienizado com álcool, foi feita a coleta da gota de sangue junto a uma lâmina de microscopia por meio de cinco leves toques desta lâmina junto à gota. Após este procedimento, a lâmina foi mantida em lugar protegido de contato com o sangue e em temperatura ambiente $\left(23^{\circ} \mathrm{C}\right)$ pelo período de nove minutos, para a efetivação do processo de coagulação do sangue colhido. Ao final deste período, o padrão morfológico destes coágulos foi microscopicamente observado sob um aumento de 40 vezes conforme estabelecido no protocolo ${ }^{18} \mathrm{e}$, a respectiva graduação qualitativa atribuída foi registrada em uma planilha eletrônica para posterior análise estatística. Como medida de padronização, a observação dos padrões morfológicos dos diferentes coágulos obtidos foi realizada por três diferentes avaliadores, que não conheciam a identificação da cada uma das lâminas de microscopia observadas, associada à avaliação computadorizada feita através do programa denominado OxyScanner elaborado e comercializado pela Aldea Global Soluciones (AGS), a qual concedeu-nos uma cópia do referido software.

Para a análise estatística dos valores dos diferentes parâmetros avaliados no presente estudo, expressos pela media \pm erro padrão da média (EPM), foi utilizado o teste de Kruskal Wallis após a verificação da normalidade. Como pós-teste para a comparação entre os pares de médias utilizou-se o teste de Dunn, sendo considerada significativa a diferença quando $\mathrm{p}<0,05$. Essas análises foram conduzidas, utilizando-se do software GraphPad InStat versão 3.00 para Windows 95 .

\section{RESULTADOS}

O esforço físico foi interrompido no momento em que os indivíduos avaliados apontaram o índice de percepção do esforço realizado equivalente ao valor 10 , de maneira que o tempo total médio de realização do referido esforço variou de 35 a 45 minutos.

Através do monitoramento da FC, observou-se que a FC média dos indivíduos em repouso foi de $77 \pm 3$ batimentos por minutos (bpm). Porém esta FC elevou-se significativamente $(\mathrm{p}<0,001)$ ao final do período de realização do esforço para um valor médio de $165 \pm 7 \mathrm{bpm}$. Embora a FC média posterior à CIC $(101 \pm 4 \mathrm{bpm})$ possa ser considerada significativamente maior $(p<0,01)$ em relação ao valor de repouso, ela representa uma significativa redução $(p<0,001)$ em relação ao valor médio adquirido ao final do esforço físico. Ao final do período de 10 minutos posteriores à $\mathrm{CIC}$, a FC média $(78 \pm 5)$ retornou ao parâmetro de repouso, visto que a mesma não se apresentou estatisticamente igual $(\mathrm{p}>0,05)$ à $\mathrm{FC}$ de repouso (Tabela 1 ).

A partir do valor médio da PA sistólica em repouso $(118 \pm 3 \mathrm{mmHg})$, observou-se uma significativa elevação 
( $p<0,001)$ ao final do esforço físico $(161 \pm 4 \mathrm{mmHg})$. Ao final da CIC, o valor observado $(101 \pm 4 \mathrm{mmHg})$ mostra-se significativamente menor $(\mathrm{p}<0,001)$ do que valor obtido anteriormente em função do esforço físico, porém este mesmo valor de PAS pós-CIC apresenta-se estatisticamente igual $(\mathrm{p}<0,05)$ ao valor de repouso, assim como o que ocorreu ao final do período de 10 minutos posteriores à CIC $(109 \pm 3)$ (Tabela 1$)$.

De acordo com os dados da Tabela 1 , as variações dos valores da PA diastólica (PAD) em repouso $(78 \pm 2 \mathrm{mmHg})$, ao final do esforço físico $(83 \pm 2)$, ao final da CIC $(77 \pm 3)$ e no décimo minuto após a CIC $(76 \pm 4)$, não foram suficientes para estabelecer diferenças significativas $(p>0,05)$ entre os mesmos.

Ao analisarmos os valores médios referentes à TC contidos na Tabela 1 , observamos que a partir do valor de repouso $\left(37 \pm 0^{\circ} \mathrm{C}\right)$ houve uma significativa elevação $(\mathrm{p}<0,001)$ ao final do esforço físico $\left(38 \pm 0^{\circ} \mathrm{C}\right)$. Porém esta elevação foi significativamente reduzida $(p<0,001)$ em função da $\mathrm{CIC}\left(37 \pm 0^{\circ} \mathrm{C}\right)$, valor este que também foi observado ao final do décimo minuto pós-CIC $\left(37 \pm 0^{\circ} \mathrm{C}\right)$.

Embora os graus do EOx atribuídos após a observação microscópica do padrão morfológico do coágulo sanguíneo tenham um caráter qualitativo, ao analisarmos os mesmos de forma quantitativa, observamos que a partir do valor médio em repouso $(1,2 \pm 0$ - grau I), houve um significativo aumento $(\mathrm{p}<0,01)$ ao final do esforço físico $(1,8 \pm 0$ - grau II). Porém, ao final da CIC, foi possível observar uma significativa redução $(\mathrm{p}<0,001)$ para o valor de $1,0 \pm 0$ (grau I), o qual pode ser considerado estatisticamente igual $(\mathrm{p}>0,05)$ ao valor de repouso. (Figura 1 ). As variações do EOx e suas correspondências quantitativas observadas em cada um dos indivíduos ao longo da realização do presente estudo estão representadas na Tabela 2.

Essas alterações do padrão morfológico do coágulo sanguíneo e seus respectivos significados qualitativos do EOx plasmático observadas microscopicamente em

Tabela 1. Valores médios \pm erro padrão da média da frequência cardíaca, das pressões arteriais sistólica e diastólica e da temperatura corporal referentes ao repouso pré-esforço, ao final do esforço e da crioimersão corporal e dez minutos de repouso após a crioimersão

$\begin{array}{lcccc} & \begin{array}{c}\text { Repouso } \\ \text { pré-esforço }\end{array} & \begin{array}{c}\text { Final do } \\ \text { esforço }\end{array} & \begin{array}{c}\text { Final } \\ \text { da CIC }\end{array} & \begin{array}{c}10 \text { minutos } \\ \text { após CIC }\end{array} \\ \text { FC }(\mathrm{bpm}) & 77 \pm 3 & 165 \pm 7^{*} & 101 \pm 4^{*} & 18 \pm 5 \\ \text { PAS }(\mathrm{mmHg}) & 118 \pm 3 & 161 \pm 4^{*} & 101 \pm 4^{*} & 109 \pm 3 \\ \text { PAD }(\mathrm{mmHg}) & 78 \pm 2 & 83 \pm 2 & 77 \pm 3 & 76 \pm 4 \\ \text { TC }\left({ }^{\circ} \mathrm{C}\right) & 37 \pm 0 & 38 \pm 0^{*} & 37 \pm 0 & 37 \pm 0\end{array}$

FC: frequência cardíaca; bpm: batida por minuto; PAS: pressão arterial sistólica; PAD: pressão arterial diastólica; TC: temperatura corporal; CIC: crioimersão corporal; \#diferença estatisticamente significativa em relação aos demais valores médios do respectivo parâmetro; *diferença significativa em relação ao valor médio de repouso, considerando ps 0,05

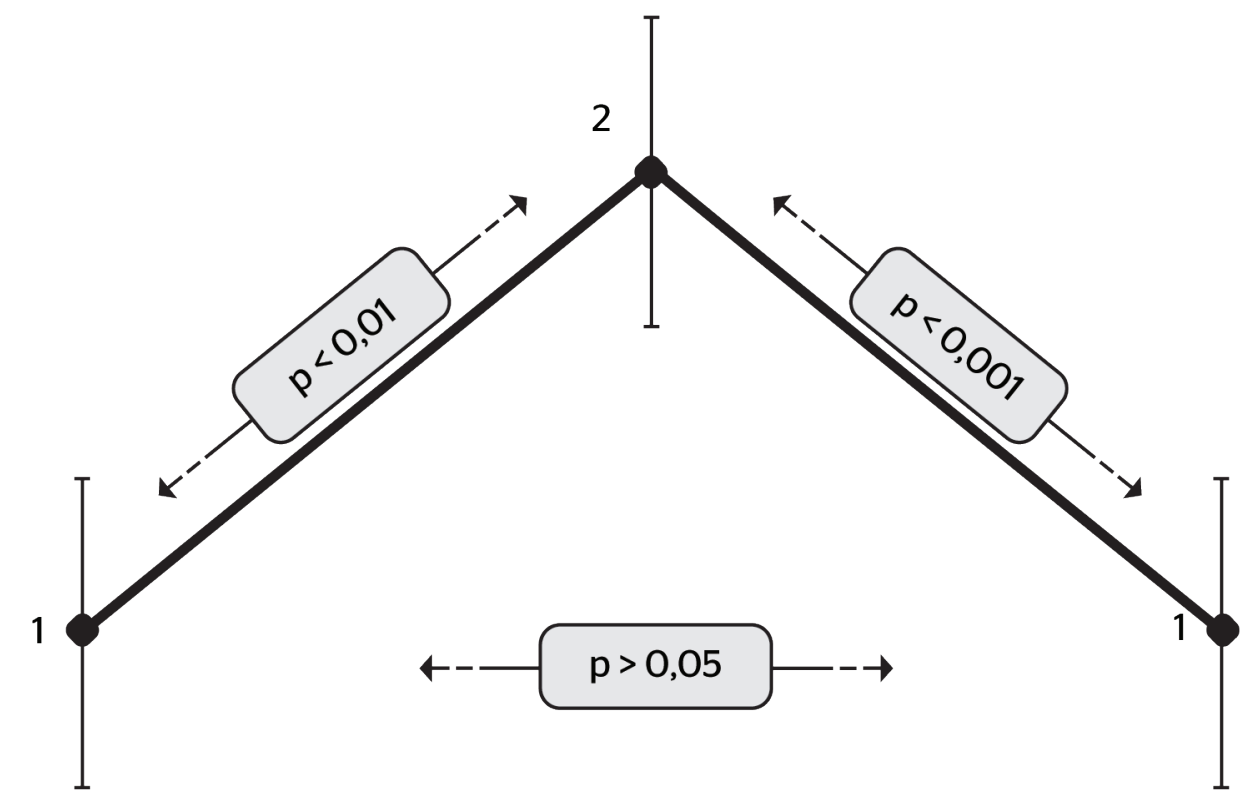


lâminas de indivíduos avaliados no presente estudo, nas situações de repouso, após o esforço físico e após a CIC, estão contidos na Figura 2.

\section{DISCUSSÃO}

Embora os danos teciduais causados pela ação oxidativa das EROs sejam mais acentuados nos organismos destreinados $^{19}$, a expressão de biomarcadores do EOx também pode ocorrer nos treinados, dependendo do esforço realizado ${ }^{20}$. Observa-se que a queda do desempenho após a realização do esforço agudo especialmente está comumente acompanhada pela elevação metabólitos da oxidação proteica e/ou da peroxidação lipídica (PL) ${ }^{21}$. Há muito, têm sido documentados aumentos significativos na concentração de malondealdeído (MDA) em decorrência da PL nos músculos esqueléticos de ratos sedentários após o esforço agudo e exaustivo ${ }^{11}$.

Tabela 2. Aspectos qualitativos individuais dos graus de variação da morfologia do estresse oxidativo e suas respectivas correspondências quantitativas em repouso, após o esforço físico e após a crioimersão corporal

\begin{tabular}{|c|c|c|c|c|c|c|}
\hline \multirow[b]{3}{*}{ Indivíduo } & \multicolumn{6}{|c|}{ Graduação do estresse oxidativo } \\
\hline & \multicolumn{3}{|c|}{ Aspectos qualitativos } & \multicolumn{3}{|c|}{$\begin{array}{l}\text { Correspondência quantitativa } \\
\text { Valores médios obtidos }\end{array}$} \\
\hline & $\mathrm{R}$ & EF & $\mathrm{ClC}$ & $\mathrm{R}$ & $\mathrm{EF}$ & $\mathrm{ClC}$ \\
\hline । & I & $\|$ & । & 1 & 2 & 1 \\
\hline$\|$ & 1 & $\|$ & । & 1 & 2 & 1 \\
\hline III & । & । & । & 1 & 1 & 1 \\
\hline IV & । & $\|$ & । & 1 & 2 & 1 \\
\hline V & $\|$ & $\|$ & 1 & 2 & 2 & 1 \\
\hline VI & 1 & 1 & । & 1 & 1 & 1 \\
\hline VII & $\|$ & $\|$ & 1 & 2 & 2 & 1 \\
\hline VIII & । & । & । & 1 & 1 & 1 \\
\hline IX & 1 & $\|$ & I & 1 & 2 & 1 \\
\hline$x$ & 1 & $\|$ & I & 1 & 2 & 1 \\
\hline$X I$ & 1 & $\|$ & 1 & 1 & 2 & 1 \\
\hline XII & 1 & $\|$ & I & 1 & 2 & 1 \\
\hline Grupo & 1 & $\|$ & 1 & $1,2 \pm 0$ & $1,8 \pm 0^{*}$ & $1,0 \pm 0$ \\
\hline
\end{tabular}

R: repouso; EF: esforço físico; CIC: crioimersão corporal; *diferença estatisticamente significativa entre os valores médios apontados em relação aos demais valores médios, considerando p 0,05

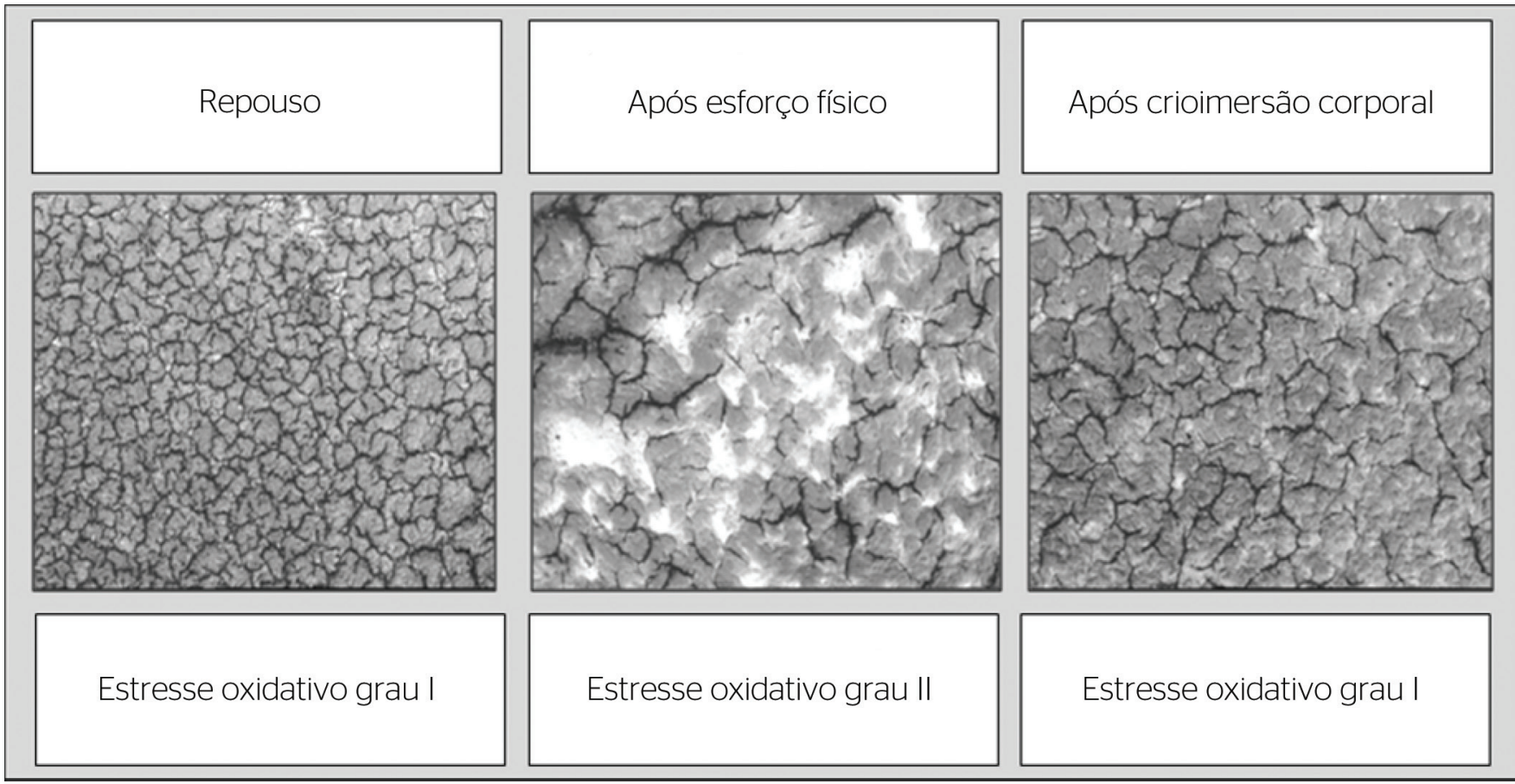

Figura 2. Imagens dos padrões morfológicos do coágulo sanguíneo e seus respectivos significados qualitativos do estresse oxidativo presente em repouso, após o esforço físico aeróbio exaustivo e após a crioimersão corporal 
Por terem vários sítios reativos ao longo de suas cadeias moleculares, as proteínas estão constantemente susceptíveis a reações de oxidação e redução ${ }^{22}$, de tal forma que no EOx induzido pelo esforço podem ocorrer danos nos seus aminoácidos após a oxidação dos antioxidantes denominados grupamentos sufidrila $(\mathrm{SH})$ livres no plasma ${ }^{23-25}$.

À medida que ERO no plasma reagem junto às fibronectinas, surgem alterações na integridade da matriz extracelular no tecido sanguíneo, provocando consequentemente alterações morfológicas dos padrões de coagulação, as quais são indicadoras específicas de distúrbios metabólicos oxidativos, pois, a contínua superfície plasmática é decorrente das ligações covalentes existentes entre as fibronectinas solúveis junto aos componentes da substância fundamental (colágeno, elastina, ácido hialurônico e sulfato de condroitina) e às membranas celulares ${ }^{18}$. Estas referidas alterações do padrão morfológico do coágulo decorrentes das ações oxidativas das ERO junto às fibrinas plasmáticas, caracterizadas pelo aumento da área total de descontinuidade da matriz extracelular em relação à área do coágulo interligada por fibrina, foram observadas no presente estudo na maioria significativa dos indivíduos no momento final do período de realização do esforço físico agudo e extenuante.

Em uma revisão sistemática realizada com o objetivo de reunir informações a respeito dos efeitos bioquímicos e fisiológicos decorrentes da CIC, evidenciou-se que a magnitude destas respostas orgânicas está intimamente relacionada ao fator aclimatação. Enquanto a $\mathrm{FC}$ sofre elevação na $\mathrm{CIC}$ a $15^{\circ} \mathrm{C}$ por até 5 minutos, na $\mathrm{CIC}$ a $10^{\circ} \mathrm{C}$ de longa duração a FC sofre uma importante redução ${ }^{26}$.

Neste referido estudo ${ }^{26}$, observou-se também que dentre as diversas modificações orgânicas induzidas pela $\mathrm{CIC}$ a $15^{\circ} \mathrm{C}$ por até 5 minutos está o EOx associado ao aumento na formação dos radicais livres, em consequência ao aumento súbito do metabolismo e da TC como mecanismo de proteção frente à aguda exposição corporal ao frio. Entretanto, tal como a redução da FC citada anteriormente, mediante o modelo de $\mathrm{CIC}$ a $10^{\circ} \mathrm{C}$ por 10 minutos, utilizado no presente estudo, foi possível observar uma redução do EOx presente ao final da realização do esforço físico agudo e exaustivo, com base na avaliação do padrão morfológico do coágulo.

As lesões dos tecidos moles são caracterizadas pelo elevado metabolismo celular, decorrente do esforço físico intenso e sem adequada recuperação ${ }^{7}$. Por isso, a propriedade de reduzir a taxa do metabolismo tissular é um dos principais objetivos crioterapêutico ${ }^{27,28}$.

No presente estudo, observou-se que a CIC realmente é um procedimento redutor da TC, visto que após a sua utilização, a significativa elevação da temperatura timpânica decorrente da elevação metabólica imposta pela demanda energética relativa ao esforço realizado, foi reduzida ao valor de repouso. Assim como foi observado em estudos recentes, nos quais a redução da TC mediante a utilização da CIC como estratégia de recuperação ${ }^{29-32}$.

A eficiência $\mathrm{CIC}$ em reduzir a $\mathrm{FC}$, como relatado em diferentes estudos ${ }^{29,30,33}$, foi novamente observa$\mathrm{da}$ neste estudo, à medida que as significativas elevações da FC e da PA, observadas ao final do esforço, foram significativamente reduzidas e igualadas aos respectivos valores de repouso, mediante a CIC imediata ao esforço físico.

Embora as evidências relativas às variações do $\mathrm{EOx}$ e da viabilidade do teste utilizado sejam relevantes, apontamos como limitações do presente estudo o uso de amostras pareadas tendo como controle os resultados obtidos nos indivíduos em repouso. Sendo assim, em função de um maior rigor metodológico em futuros estudos, recomenda-se que no delineamento experimental seja contemplado um grupo controle que não seja submetido aos ensaios experimentais.

\section{CONCLUSÃO}

Embora sejam necessários mais estudos científicos com o teste de MEOP, concluímos que para o presente estudo o referido teste mostrou-se viável. Os dados encontrados no presente estudo sugerem que a CIC em água a $10^{\circ} \mathrm{C}$ por 10 minutos após o esforço físico agudo e exaustivo apresenta-se como uma importante conduta fisioterapêutica para a redução do EOx após o esforço físico.

\section{AGRADECIMENTO}

Ao Sr. Carlos Bonilla da empresa Aldea Global Soluciones (AGS) pelo fornecimento do sistema de avaliação do estresse oxidativo - OxyScanner. 


\section{REFERÊNCIAS}

1. Diffe GM, Chung E. Altered single cell force-velocity and Power properties in exercise-trained rat myiocardium. J Appl Physiol. 2003;94(5):1941-8

2. Matsudo VKR, Matsudo SMM. Evidências da importância da atividade física nas doenças cardiovasculares e na saúde. Diagn Tratamento. 2000;5(2):10-7.

3. Lees SJ, Booth FW. Physical inactivity is a disease. World Rev Nutr Diet. 2005;95:73-9

4. Booth FW, Chakravarthy MV, Gordon SE, Spangenburg EE. Waging war on physical inactivity: using modern molecular ammunition against an ancient enemy. J Appl Physiol. 2002;93(1):3-30.

5. Volek J S, Kraemer WJ, Rubin MR, Gómez AL, Ratamess NA, Gaynor P. L-Carnitine L-tartrate supplementation favorably affects markers of recovery from exercise stress. Am J Physiol Endocrinol Metab. 2002;282(2):E474-82.

6. Rondon MUPB, Alves MJNN, Braga AMFW, Negrão CE. Exercício físico e insuficiência cardíaca. Rev Soc Cardiol Estado de São Paulo. 2000,10(1):153-60.

7. Araujo GG, Gobatto CA, Hirata RDC, Hirata MH, Cavaglieri CR, Verlengia R. Respostas fisiológicas para detectar o overtraining. R da Educação Física/UEM. 2008:19(2):275-89.

8. Finaud J, Lac G, Filaire E. Oxidative stress: relationship with exercise and training. Sports Med. 2006;36(4):327-58.

9. Gomez-Cabrera M, Borrás C, Pallardó FV, Sastre J, Ji LL, Viña J. Decreasing xanthine oxidase-mediated oxidative stress prevents useful cellular adaptations to exercise in rats. J Physiol. 2005;567(Pt 1):113-20.

10. McArdle A, Vasilaki A, Jackson M. Exercise and skeletal muscle ageing cellular and molecular mechanisms. Ageing Res Rev. 2002;(1):79-93.

11. Bachur JA, Garcia SB, Vannucchi H, Jordão AA, Chiarello PG, Zucoloto S. Anti-oxidative systems in rat skeletal muscle after acute physical exercise. Appl Physiol Nutr Metab. 2007;32(2):190-6.

12. Smolka M, Zoppi C, Alves AA, Silveira LR, Marangoni S, Pereira-DaSilva L, et al. HSP72 as a complementary protection against oxidative stress induced by exercise in the soleus muscle of rats. Am J Physiol Regul Integr Comp Physiol. 2000;279(5):R1539-45.

13. Cochrane DJ. Alternating hot and cold water immersion for athlete recovery: a review. Physical Therapy in Sport. 2004;5:26-32.

14. Wilcock IM, Cronin JB, Hing WA. Physiological response to water immersion: a method for sport recovery? Sports Med. 2006;36(9):747-65

15. Vaile J, Halson S, Gill N, Dawson B. Effect of cold water immersion on repeat cycling performance and thermoregulation in the heat. J Sports Sci. 2008;26(5):431-40

16. Monteiro W. Simão R, Farinatti P. Manipulação na ordem dos exercícios e sua influência sobre o número de repetições e percepção subjetiva de esforço em mulheres treinadas. Rev Bras Med Esporte. 2005;11(2):146-50.

17. Bachur JA, Quemelo PR, Bachur CAK, Domenciano JC, Martins CHG, Stoppa MA, et al. Avaliação do efeito da hipotermia por crioimersão corporal, nos neutrófilos e linfócitos sanguíneos de ratos submetidos ao exercício físico agudo. Rev Bras Hematol Hemoter. 2008:30(6):470-4

18. Olszewer E. Microscopia ótica como método de medida de radicais livres. 2a ed. - São Paulo:Icone, 2001

19. Lamprecht M, Greilberger J, Oetti K. Analytical aspects of oxidatively modified substances in sports and exercises. Nutrition. 2004;20(7-8):728-30.

20. Antunes-Neto JMF, Pilatti LS, Filho JPA, Magalhães NP. Cinética de marcadores de estresses oxidativo e fisiológico em condição de corrida exaustiva. Revista Brasileira de Educação Física, Esporte, Lazer e Dança. 2007;2(2):56-68.

21. Bloomer RJ. The role of nutritional supplements in the prevention and treatment of resistance exercise-induced skeletal muscle injury. Sports Med. 2007;37(6):519-32

22. Ascenção A, Rebelo A, Oliveira E, Marques F, Pereira L, Magalhães J. Biochemical impact of a soccer match - analysis of oxidative stress and muscle damage markers throughout recovery. Clin Biochem. 2008;41(10-11):841-51.

23. Chevion M, Berenshtein E, Stadtman ER. Human studies related to protein oxidation: protein carbonyl content as a marker of damage. Free Radic Res. 2000;33 Suppl:99-108

24. Nikolaidis MG, Jamurtas AZ, Paschalis V. Fatouros IG, Koutedakis $Y$, Kouretas D. The effect of muscle-damaging exercise on blood and skeletal muscle oxidative stress: magnitude and time-course considerations. Sports Med. 2008:38(7):579-606

25. Barreiros ALBS, David JM, David JP. Estresse oxidativo: relação entre geração de espécies reativas e defesa do organismo. Quím Nova. 2006;29(1):113-23

26. Bleakley CM, Davison GW. What is the biochemical and physiological rationale for using cold-water immersion in sports recovery? A systematic review. Br J Sports Med. 2010;44(3):179-87.

27. Barnett A. Using recovery modalities between training sessions in elite athletes: does it help? Sports Medicine. 2006;36(9):781-96.

28. Oliveira NML, Rainero EP, Salvini TF. Three intermittent sessions of cryotherapy reduce the secondary muscle injury in skeletal muscle of rat. J Sports Sci Med. 2006;5(2):228-34

29. Pointon M, Duffield R, Cannon J, Marino FE. Cold water immersion recovery following intermittent-sprint exercise in the heat. Eur J Appl Physiol. 2012;112(7):2483-94

30. DeMartini JK, Ranalli GF, Casa DJ, Lopez RM, Ganio MS, Stearns RL, McDermott BP, Armstrong LE, Maresh CM. Comparison of body cooling methods on physiological and perceptual measures of mildly hyperthermic athletes. J Strength Cond Res. 2011;25(8):2065-74.

31. Peiffer JJ, Abbiss CR, Watson G, Nosaka K, Laursen PB. Effect of a 5-min cold water immersion recovery on exercise performance in the heat. Br J Sports Med. 2010;44(6):461-5.

32. Peiffer JJ, Abbiss CR, Watson G, Nosaka K, Laursen PB. Effect of cold water immersion after exercise in the heat on muscle function, body temperatures, and vessel diameter. J Sci Med Sport. 2009;12(1):91-6.

33. Faria AFJS, Queiroz LL. Influência do resfriamento e do aquecimento local na flexibilidade dos músculos isquiotibiais. Rev Bras Fisioter. 2007;11(1):57-61. 